\title{
A pilot survey of ventilated cancer patients' perspectives and recollections of early mobility in the intensive care unit
}

\author{
Steven H. Hsu ${ }^{1,2}$ (D) Claudine Campbell ${ }^{3}$ - Amanda K. Weeks ${ }^{3}$ - Maryann Herklotz ${ }^{3}$. Natalie Kostelecky ${ }^{1}$. \\ Stephen M. Pastores ${ }^{1} \cdot$ Neil A. Halpern ${ }^{1} \cdot$ Louis P. Voigt $^{1}$
}

Received: 12 November 2018 / Accepted: 9 May 2019 / Published online: 29 May 2019

(C) Springer-Verlag GmbH Germany, part of Springer Nature 2019

\begin{abstract}
Purpose To determine the level of recall, satisfaction, and perceived benefits of early mobility (EM) among ventilated cancer patients after extubation in the intensive care unit (ICU).

Methods A survey of patients' perceptions and recollections of EM was administered within $72 \mathrm{~h}$ of extubation. Data on recall of EM participation, activities achieved, adequacy of staffing and rest periods, strength to participate, activity level of difficulty, satisfaction with staff instructions, breathing management, and overall rating of the experience were analyzed. The Confusion Assessment Method for ICU (CAM-ICU) was used for delirium screening.

Results Fifty-four patients comprised the study group. Nearly $90 \%$ reported satisfaction with instructions, staffing, rest periods, and breathing management during EM. Participants indicated that EM maintained their strength $(67 \%)$ and gave them control over their recovery $(61 \%)$; a minority felt optimistic $(37 \%)$ and safe $(22 \%)$. Patients who achieved more sessions and "out-of-bed" exercises had better recall of actual activities compared with those who exercised in bed. Overall, patients with CAM-ICU-positive results (33\%) performed less physical and occupational therapy exercises.

Conclusions Ventilated cancer patients reported an overall positive EM experience, but factual memory impairment of EM activities was common. These findings highlight the needs and the importance of shaping strategies to deliver a more patient focused EM experience.
\end{abstract}

Keywords Early mobility $\cdot$ Mechanical ventilation $\cdot$ Satisfaction $\cdot$ Perception $\cdot$ Memory recall

This article was presented in part, as an abstract at the Society of Critical Care Medicine Critical Care Congress in Orlando, FL on February 23, 2016.

Electronic supplementary material The online version of this article (https://doi.org/10.1007/s00520-019-04867-1) contains supplementary material, which is available to authorized users.

Louis P. Voigt

voigtl@mskcc.org

1 Critical Care Center, Department of Anesthesiology and Critical Care Medicine, Memorial Sloan Kettering Cancer Center, 1275 York Ave, Suite C-1179, New York, NY 10065, USA

2 Department of Medicine, Houston Methodist Hospital, Houston, TX, USA

3 Department of Neurology and Rehabilitation Medicine, Memorial Sloan Kettering Cancer Center, New York, NY, USA

\section{Introduction}

Early mobility (EM) in the intensive care unit (ICU) involves the initiation of combined physical therapy (PT) and occupational therapy (OT) activities in mechanically ventilated patients during the first $2-5$ days of ICU admission [1,2]. Patients undergoing EM face a multitude of emotional, cognitive and physical challenges: cognitive impairment affecting their ability to follow directions, generalized weakness, and their attachment to monitoring devices [3,4]. Nevertheless, even in this setting, early mobility has been demonstrated to attenuate ICUacquired weakness, improve functional recovery, reduce the incidence and duration of delirium, ventilator days and ICU and hospital length of stay (LOS), and decrease hospital costs $[2,5,6]$. 
Despite the established benefits of EM, barriers exist at multiple levels, including health care providers' competence and knowledge of the risks and benefits of EM, patients' anxiety and fear, and adequate administrative support for comprehensive EM programming. Critically ill patients with cancer experience additional challenges, resulting from disease symptoms and treatment-related side effects, which adversely impacts their quality of life [7]. Previous studies of EM in critically ill patients mostly derived from the perspective of health care providers and have focused primarily on its safety, feasibility, barriers, and perceived benefits [8-11]. A few studies have addressed patient and family perceptions of physical therapy, a key component of EM; however, these studies were limited to patients in the outpatient arena or focused on survivors of critical illness [12-14]. Only one study has dealt with patient and family perceptions of PT in the ICU. Participants in this study completed a survey at the end of 28 days of PT or at hospital discharge [15]. Interpreting such a study is problematic because the factual memory of patients recalling ICU stay events varies greatly during and after critical illness [16].

Understanding patients' perceptions and recollections of EM during mechanical ventilation in the ICU may help clinicians better understand patients' perspectives regarding these activities. Such awareness may lead to interventions that will facilitate patients' participation in EM and enrich their experience. The primary objectives of this pilot study were to determine the level of recall, satisfaction, and perceived benefits of EM recipients shortly after extubation in the ICU. Additionally, as secondary end-points, we analyzed the degree of correlation between the PT and OT exercises recalled by the patients and the actual activities performed.

\section{Methods}

The study was conducted in the 20-bed adult medical-surgical ICU at Memorial Sloan Kettering Cancer Center, New York, NY, from October 2014 to April 2016. The ICU is staffed by intensivist-run multidisciplinary teams [registered nurses $(\mathrm{RN})$, respiratory therapists (RT), pharmacists, nutritionists, social workers, nurse practitioners (NPs), physician assistants, anesthesiology residents, and critical care fellows)], and dedicated rehabilitation team of 3 physical therapists (PT) and 2 occupational therapists (OT). Each patient EM session is staffed by one RT, one PT, one OT, and the bedside RN.

At our center, all patients receiving mechanical ventilation are assessed for EM participation within $48 \mathrm{~h}$ of intubation. Eligible patients for EM are identified by the bedside RN, RT, PT, and OT staff during their early morning rounds on weekdays. Patients exhibiting severe altered mental status, elevated intracranial pressure, non-secure or difficult airway, active myocardial ischemia, hemodynamic instability, active gastrointestinal blood loss, or open abdomen are excluded. All potential EM patients are cleared and approved by the intensivist-led ICU teams. Once cleared, the patients undergo a comprehensive assessment followed by a series of physical and cognitive EM exercises as determined and instructed by the PT and OT staff. These activities include the following: (1) in bed activities such as passive and active range of motion exercises; (2) use of iPad in bed; (3) seated edge of bed activities including self-care, postural correction/control, and active range of motion; (4) sit to stand, and transfer training to chair or commode; and (5) standing exercises and gait training. The bedside RN, RT, and other adjunctive staff monitor the patients' vital signs, cardiovascular and respiratory status, level of comfort, and tolerance to these activities [7]. In addition, they mitigate mishaps by securing endotracheal, nasogastric and/or thoracic tubes, as well as abdominal/pelvic, urinary and/or vascular catheters.

\section{Survey development and administration}

A 12-question survey (Supplement 1) addressing the recollections and perceptions of EM recipients was developed by a multidisciplinary group (psychiatrists, nurse educators, PTs, OTs, RNs, NPs, and critical care physicians). Components from the Family Satisfaction in the ICU (FS-ICU) and ICU Memory Tool (ICUMT) were included [17, 18], specifically items derived from the sections on symptom management and how patients were treated. The survey was reviewed by the Patient and Caregiver committee for readability at 7 th to 8 th grade reading level per institution guidelines [19]. A pilot of the survey was administered to 5 patients, who provided the following feedback: (1) the questions were easy to read and understand; (2) questions eliciting patient feelings were welcomed [questions 9-12]; and (3) a simplified definition for early mobility was requested. Recommendations from the pilot were incorporated into the final version of the survey, which was approved by the Institutional Review Board (IRB). Informed consent was obtained from all study patients.

Immediate factual memory assessment has been shown to have the highest yield of factual recall within $72 \mathrm{~h}$ of a patient's ICU stay [20]. Thus, the survey was administered within 24 to $72 \mathrm{~h}$ of extubation to all EM recipients who agreed to participate and remained extubated at the time of survey. CAM-ICU was screened on the day of survey prior to its administration. If the patient completed the survey by responding that they had no EM recollection, they were subsequently excluded from analysis. We also excluded patients who missed the 72-h window of survey administration, declined to answer questions, were discharged from the hospital, or expired within $48 \mathrm{~h}$ of extubation. Additionally, patients who exhibited signs of medical or mental instability were reintubated, or continuously required mechanical ventilation were excluded. 
The survey (Supplement 1) focused on patients' recall of participation in EM and PT/OT activities achieved, adequacy of staffing and rest periods, strength to participate in EM, level of difficulty of EM activities, satisfaction with staff instructions, management of respiratory support, and overall rating of the EM experience. Three survey questions (5, 6, and 11) used a multi-answer with a cumulative total of $>100 \%$ (see Supplement 1). Actual individual EM activities achieved, as derived from the PT and OT assessment notes within the electronic medical record, were compared with those recorded on the survey. To our knowledge, there is no quantitative or percentage of factual recall established for positive correlation with ICU patient outcome. Thus, we arbitrarily applied a $\geq 50 \%$ correlation to define positive recall of EM activities.

Data on demographics (age and gender), Mortality Probability Model (MPM) II severity of illness score on ICU admission, admitting service (medical or surgical), primary ICU admitting diagnoses, cancer type, history of dementia or cognitive impairment, delirium screen on the day of the survey, and outcomes (ventilator days, ICU and hospital LOS, discharge destinations, and ICU and hospital mortality rates) and EM-related data were collected for each survey participant. EM specific data differentiated between two levels of physical activities: "bed-level exercises" (i.e., arm and leg exercises and sitting at the edge of the ICU bed) and "out-ofbed" activities (i.e., standing by the side of the ICU bed, sitting in a chair, walking inside/outside the ICU room, and washing face/body). Actual activities were recorded and documented in PT/OT daily progress notes entered in patient's electronic record. The survey also queried cognitive interventions (reading, writing, filling calendar, using communication device, and memory exercises). Subgroup analysis was performed based on CAM-ICU results.

Demographic and clinical characteristic results are presented as mean \pm standard deviation (SD), median and interquartile ranges, absolute numbers, and/or percentages. The data were analyzed using Fischer's exact test for categorical variables and Student's $t$ test or Wilcoxon rank-sum test for continuous variables. A $p$ value of $<0.05$ was considered significant. Statistical analyses were performed using GraphPad Prism 7.0 (GraphPad Software, San Diego, CA, USA).

\section{Results}

Between October 2014 and April 2016, 601 unique MV patients were identified. A total of $218(40 \%)$ patients received EM. One hundred and forty-eight (68\%) patients were excluded (Fig. 1) and 70 (30\%) EM recipients completed the survey. Of these, 16 participants indicated on the survey that they did not recall participation in EM resulting in 54 evaluable patients for analysis.

\section{Patient characteristics}

The mean age of the study patients was 62.5 years and $61 \%$ were male. Mean MPM II was 43, 56\% were medical patients and $65 \%$ had a solid tumor (Table 1 ). The primary reason for ICU admission was respiratory failure in $61 \%$ of cases. The median duration on mechanical ventilation was 5 days (3.07.0), and ICU and hospital LOS were 11.5 (8.0-18.8) and 34.5 (22-51) days, respectively. The median time from extubation to survey was 1 day (1-3). One-third (33\%) of patients

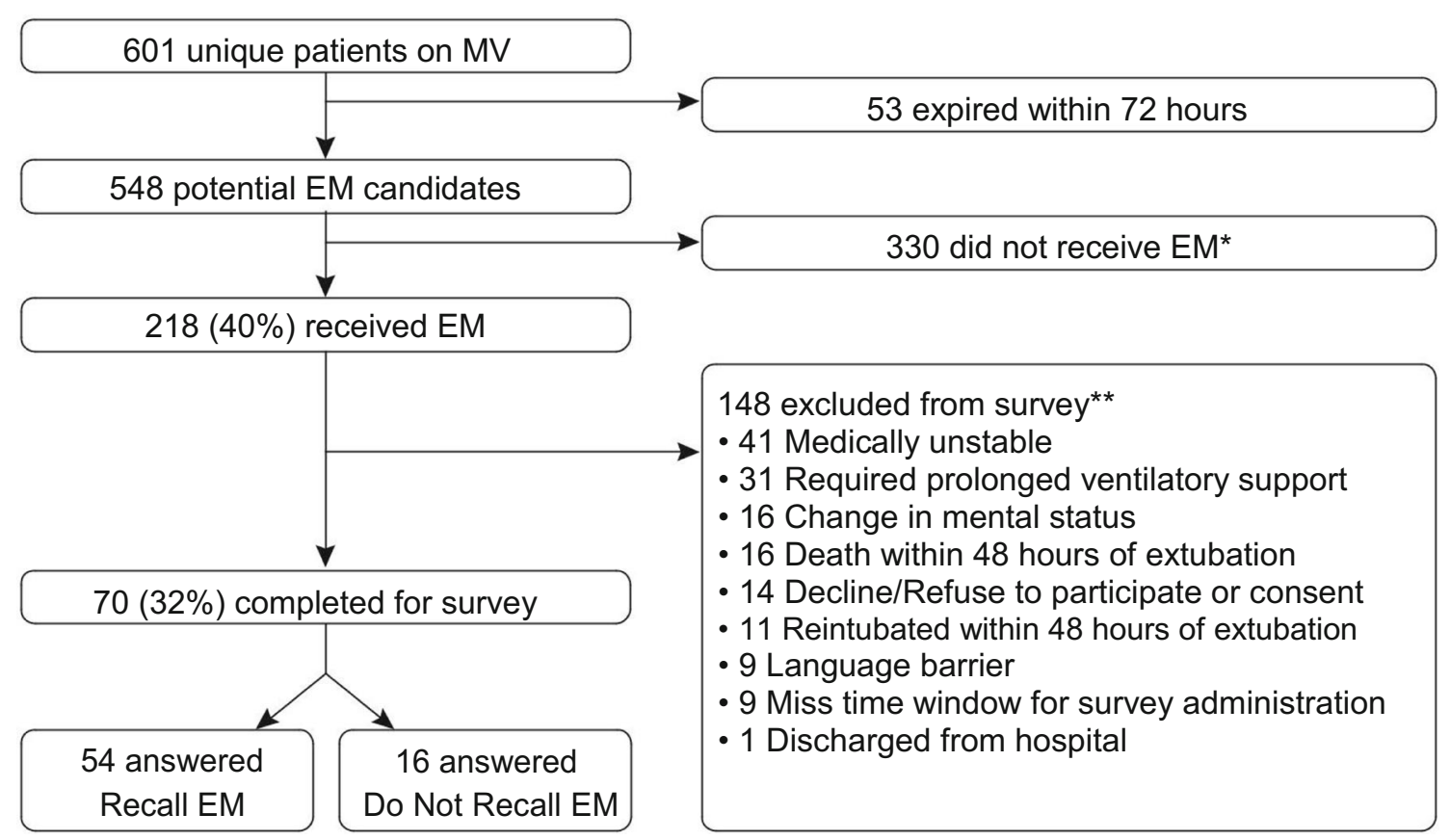

Fig. 1 Flowchart of patients included in early mobility (EM) survey 
Table 1 Demographic and clinical characteristics $(n=54)$

\begin{tabular}{ll} 
Age, years (mean \pm SD) & $62.5 \pm 10.4$ \\
Male $(n, \%)$ & $33(61 \%)$ \\
MPM II 0 score on ICU admission (mean \pm SD) & $43 \pm 19.2$ \\
Medicine service $(n, \%)$ & $30(56 \%)$ \\
Cancer type $(n, \%)$ & \\
Solid tumor & $35(65 \%)$ \\
Hematologic malignancy & $16(30 \%)$ \\
Other & $3(5 \%)$ \\
Primary diagnosis $(n, \%)$ & \\
Respiratory failure & $33(61 \%)$ \\
Sepsis & $8(15 \%)$ \\
Other & $13(24 \%)$ \\
Ventilator days (median, IQR) & $5.0(3-7)$ \\
ICU LOS, days (median, IQR) & $11(8-18.8)$ \\
Hospital LOS, days (median, IQR) & $34(22-51)$ \\
ICU mortality $(n, \%)$ & $4(7 \%)$ \\
Hospital mortality $(n, \%)$ & $17(31 \%)$ \\
Intubation to first EM session (median, IQR) & $1(1-5)$ \\
Extubation to survey (median, IQR) & $1(1-3)$ \\
PT sessions (mean \pm SD) & $2 \pm 1$ \\
OT sessions (mean \pm SD) & $2 \pm 2$ \\
\hline
\end{tabular}

$M P M I I_{0}$, Mortality Probability Models $\mathrm{II}_{0}$ score; $I C U$, intensive care unit; $S D$, standard deviation; $I Q R$, interquartile range; $L O S$, length of stay

screened positive for delirium, and no prior history of dementia or cognitive impairment was identified for any patients. Four patients died in the ICU and 17 more died in the hospital following ICU discharge. The mean number of EM sessions per patient was $2 \pm 1$.

\section{Patient satisfaction, recall, and perceptions}

Over $80 \%$ of patients were satisfied with staff instructions and breathing management (Table 2). Similarly, over $85 \%$ of patients recalled that there were adequate staffing and rest periods. Close to two-thirds of patients reported they felt strong enough to participate and indicated the EM session's intensity was "just right." A broad range of emotions toward EM sessions were expressed with the highest percentage of patients feeling optimistic $(37 \%)$ and safe $(22 \%)$, and the others feeling scared (24\%) and frustrated (9\%). Most patients reported that EM helped them (a) maintain their strength $(67 \%)$, (b) gain control of their recovery $(61 \%)$, and (c) think more clearly (52\%).

\section{Memory recall}

In terms of recalled PT activities, patients significantly overestimated their experience of sitting in a chair, walking inside and outside of ICU room (Table 3). Regarding OT activities, the patients significantly underestimated writing
Table 2 Patient satisfaction and perceptions $(n, \%)$

\begin{tabular}{ll} 
Satisfaction level & \\
Staff instructions & $49(91 \%)$ \\
Breathing management & $45(83 \%)$ \\
Perceptions of activities & \\
Adequate staffing & $48(89 \%)$ \\
Allow to rest during EM & $50(93 \%)$ \\
Strong enough to participate & $35(65 \%)$ \\
EM sessions were just right & $33(61 \%)$ \\
Overall feelings towards EM & \\
Optimistic & $20(37 \%)$ \\
Scared & $9(24 \%)$ \\
Safe & $12(22 \%)$ \\
Frustrated & $5(9 \%)$ \\
Personal perceptions* & \\
Maintain my strength & $36(67 \%)$ \\
Give me control of recovery & $33(61 \%)$ \\
Think more clearly & $28(52 \%)$ \\
Decrease pain & $11(20 \%)$ \\
\hline
\end{tabular}

*The patients were able to select $>1$ response on the survey

and memory activities. A positive association was observed between the "level" of PT performed by patients and their degree of recalled exercises (equal or greater than 50\%). A significantly higher degree of recall was associated with outof-bed activities (greater level of mobility) compared with bed level exercises [29 (82\%) vs. $6(32 \%), p=0.0003$ for PT and $26(76 \%)$ vs. $9(45 \%), p=0.039$ for OT] (Table 4$)$. Significantly better recall was noted among patients who received at least 2.3 EM sessions.

Interestingly, in subgroup analysis, the majority of CAMICU-positive patients and about half of non-delirious patients reported feeling strong enough to participate in EM, a difference that was significant ( $89 \%$ vs $53 \%, p=0.014)$. Both CAMICU-positive and -negative patients reported similar level of recall for certain PT activities (e.g., sitting at the edge of bed $94 \%$ vs $78 \%, p=\mathrm{NS}$; sitting in chair $56 \%$ vs $56 \%, p=\mathrm{NS}$ ) and OT activities (e.g., reading $17 \%$ vs $14 \%, p=\mathrm{NS}$; using communication device $22 \%$ vs $28 \%, p=\mathrm{NS})$. However, the reality was quite different. Delirious patients performed less actual PT activities: standing by the edge of bed $(39 \%$ vs $78 \%, p=$ $0.007)$, sitting in chair ( $0 \%$ vs $36 \%, p=0.002)$, walking inside ICU room $(0 \%$ vs $31 \%, p=0.01)$; they also achieved less actual OT activity such as washing their face (6\% vs $42 \%, p=$ $0.01)$ and writing ( $44 \%$ vs. $83 \%, p=0.005)$.

\section{Discussion}

Our study is the first to describe the perceptions, range of emotions, and recall of a cohort of ventilated cancer patients 
Table 3 Recall versus actual activities

\begin{tabular}{llll}
\hline & $\begin{array}{l}\text { Remembered } \\
(n, \%)\end{array}$ & $\begin{array}{l}\text { Actual } \\
(n, \%)\end{array}$ & p value \\
\hline PT activities & & & \\
Sitting at the edge of ICU bed & $45(83 \%)$ & $45(83 \%)$ & 1.0 \\
Standing at the edge of ICU bed & $39(72 \%)$ & $34(63 \%)$ & 0.41 \\
Sitting in a chair & $30(56 \%)$ & $12(22 \%)$ & 0.0007 \\
Walking inside ICU room & $20(37 \%)$ & $10(19 \%)$ & 0.05 \\
Walking outside ICU room & $9(17 \%)$ & $2(4 \%)$ & 0.05 \\
Arm exercise & $29(54 \%)$ & $23(43 \%)$ & 0.34 \\
Leg exercise & $40(74 \%)$ & $30(56 \%)$ & 0.07 \\
Wash face/body & $17(31 \%)$ & $15(28 \%)$ & 0.83 \\
OT activities & & & \\
Reading & $9(17 \%)$ & $6(11 \%)$ & 0.58 \\
Writing & $24(44 \%)$ & $38(70 \%)$ & 0.01 \\
Memory activities & $33(61 \%)$ & $50(93 \%)$ & 0.0002 \\
Filling out a calendar & $5(9 \%)$ & $0(0 \%)$ & 0.06 \\
Use communication device & $14(26 \%)$ & $21(39 \%)$ & 0.22 \\
\hline
\end{tabular}

$P T$, physical therapy; $O T$, occupational therapy

who underwent EM and were surveyed within 24 to $72 \mathrm{~h}$ of extubation. Previous studies conducted after ICU and/or hospital discharge demonstrated factual recall decline and delusional memory retention $[18,19]$ which may negatively impact accurate memory recollection. Thus, we chose the 72-h post-extubation window to capture maximal factual recall and minimize potential recall difficulty or bias. Our results indicated that recollections were inconsistent, similar to prior ICU studies of recall $[16,21]$.

By matching the responses of survey participants to the actual EM activities they performed, we determined a more accurate level of recall. Interestingly, no statistically significant difference was detected in the degree of recall for PT and
OT activities between CAM-ICU-positive and -negative groups; yet, non-delirious patients tended to achieve better physical and cognitive performances compared with CAMICU-positive patients. Our study also demonstrates a positive association between (a) recall of the number of EM sessions completed and (b) the ability of patients to get out of bed. Such a relation suggests that repetition and advanced mobility may play a role in better memory recall.

Our study highlights the many positive and negative emotions experienced by the respondents. Slightly more than a third of the patients were optimistic about EM, while less than a quarter conveyed feeling either safe or scared. The distressing sentiments toward EM seem consistent with previous findings that critically ill patients are overwhelmed by the complexity and uncertainty of their diagnosis, management decisions, and eventual outcomes [22]. However, our data should strongly encourage the ICU clinicians who are vested in EM to be more attentive to these predicaments [8, 23, 24]. Perhaps the EM team should routinely take the emotional "temperature" of their patients in real-time to identify and address their feelings and needs.

This pilot study provides insight into patients' perceptions, satisfaction, and performance during EM, as well as the fluctuation of retention and awareness many experience. While the overall nature of critical illness is certainly devastating, we also found that most patients expressed satisfaction with the EM sessions. Respondents also indicated feeling "in control" of their recovery and "thinking more clearly" because of their participation in EM activities. These positive responses highlight the value of clear communication, patient engagement, optimal preparation, adequate staffing, provision of intermittent rest periods of care, and attention to the EM process [25]. Focus on patients' emotions and insights before, during, and after EM may help calibrate interventions in the ICU to reassure

Table 4 Characteristics of level of activities achieved during EM and degree of their recall by survey respondents

\begin{tabular}{llll}
\hline Level or range of activities achieved during EM & Recall $\geq 50 \%$ & Recall $\leq 50 \%$ & $p$ value \\
\hline & Physical therapy (PT) & $n=19$ & 13 \\
& $n=35$ & 6 & 0.0003 \\
No activities up to sitting at the edge of bed & 6 & & $n=20$ \\
Standing or higher level out-of-bed activities & 29 & 11 & 9 \\
& Occupational therapy (OT) & $1.5 \pm 0.7$ \\
No activities up to sitting at the edge of bed & $n=34$ & $1.5 \pm 0.6$ \\
Standing or higher level out-of-bed activities & 8 & 0.037 \\
EM session (PT), mean \pm SD & 26 & $2.4 \pm 1.3$ & 0.01 \\
EM session (OT), mean \pm SD & $2.3 \pm 1.3$ & 0.01 \\
\hline
\end{tabular}

A positive association was noted between the degrees of recall (equal or greater than 50\% matched) and the level of PT performed during EM. A significantly higher degree of recall was associated with "out-of-bed activities" compared with bed level exercises $(29(82 \%)$ vs. $6(32 \%), p=0.0003$ for PT and $26(76 \%)$ vs. $9(45 \%), p=0.037$ for OT). Also, a significantly better recall was noted among patients who received at least 2.3 EM sessions 
patients on the safety, quality, and support to mitigate distress and pain.

Our study has several limitations. First, the survey was administered to a relatively small cohort of patients with cancer at a single center that supports EM with appropriate resources; thus, its applicability may be limited in underresourced, non-oncologic centers. Second, only $25 \%$ of all eligible patients were able to complete the survey which limits its generalizability. Third, the survey was only piloted for readability and comprehension on a small number of patients and not tested for reliability, content validity, or clinical utility prior to implementation.

\section{Conclusions}

Our survey of eligible patients with cancer who underwent early mobility during mechanical ventilation in the ICU suggests an overall positive experience but indicates that impairment in factual memory of EM activities is common. These findings underscore the need for health care providers to carefully consider subtle hurdles to patients' participation and satisfaction with EM, and in shaping alternative strategies to deliver a richer and more patient-focused experience. Future EM program development should consider tailoring EM sessions by both PT and OT specialists based on both patients' physical and cognitive status, and particularly increasing the number of sessions and the intensity of rehabilitation activities for delirious patients.

Financial support Supported, in part, by the Core Grant (P30 CA008748) and the Department of Anesthesiology and Critical Care Medicine, Memorial Sloan Kettering Cancer Center.

Author contributions All the listed authors were involved in the study design, data collection, analysis and interpretation, and manuscript drafting. Critical manuscript revision was performed by L.P.V., S.M.P., and N.A.H., and final manuscript approval was provided by all authors.

\section{Compliance with ethical standards}

Recommendations from the pilot were incorporated into the final version of the survey, which was approved by the Institutional Review Board (IRB). Informed consent was obtained from all study patients.

Conflict of interest The authors declare that they have no conflict of interest.

\section{References}

1. Hodgson CL, Berney S, Harrold M, Saxena M, Bellomo R (2013) Clinical review: early patient mobilization in the ICU. Crit Care 17(1):207. https://doi.org/10.1186/cc11820

2. Schweickert WD, Pohlman MC, Pohlman AS, Nigos C, Pawlik AJ, Esbrook CL, Spears L, Miller M, Franczyk M, Deprizio D, Schmidt
GA, Bowman A, Barr R, McCallister KE, Hall JB, Kress JP (2009) Early physical and occupational therapy in mechanically ventilated, critically ill patients: a randomised controlled trial. Lancet 373(9678):1874-1882. https://doi.org/10.1016/S0140-6736(09) 60658-9

3. Needham DM, Korupolu R, Zanni JM, Pradhan P, Colantuoni E, Palmer JB, Brower RG, Fan E (2010) Early physical medicine and rehabilitation for patients with acute respiratory failure: a quality improvement project. Arch Phys Med Rehabil 91(4):536-542. https://doi.org/10.1016/j.apmr.2010.01.002

4. Davidson JE, Harvey MA, Bemis-Dougherty A, Smith JM, Hopkins RO (2013) Implementation of the pain, agitation, and delirium clinical practice guidelines and promoting patient mobility to prevent post-intensive care syndrome. Crit Care Med 41(9 Suppl 1):S136-S145. https://doi.org/10.1097/CCM.0b013e3182a24105

5. Lord RK, Mayhew CR, Korupolu R, Mantheiy EC, Friedman MA, Palmer JB, Needham DM (2013) ICU early physical rehabilitation programs: financial modeling of cost savings. Crit Care Med 41(3): 717-724. https://doi.org/10.1097/CCM.0b013e3182711de2

6. Hodgson CL, Bailey M, Bellomo R, Berney S, Buhr H, Denehy L, Gabbe B, Harrold M, Higgins A, Iwashyna TJ, Papworth R, Parke R, Patman S, Presneill J, Saxena M, Skinner E, Tipping C, Young P, Webb S, Trial of Early A, Mobilization Study I (2016) A binational multicenter pilot feasibility randomized controlled trial of early goal-directed mobilization in the ICU. Crit Care Med 44(6):11451152. https://doi.org/10.1097/CCM.0000000000001643

7. Weeks A, Campbell C, Rajendram P, Shi W, Voigt L (2017) A descriptive report of early mobilization for critically ill ventilated patients with cancer. Rehabil Oncol 35(3):144-150. https://doi.org/ 10.1097/01.REO.0000000000000070

8. Dubb R, Nydahl P, Hermes C, Schwabbauer N, Toonstra A, Parker AM, Kaltwasser A, Needham DM (2016) Barriers and strategies for early mobilization of patients in intensive care units. Ann Am Thorac Soc 13(5):724-730. https://doi.org/10.1513/AnnalsATS. 201509-586CME

9. Castro E, Turcinovic M, Platz J, Law I (2015) Early mobilization: changing the mindset. Crit Care Nurse 35(4):e1-5; quiz e6. https:// doi.org/10.4037/cen2015512

10. Parry SM, Knight LD, Connolly B, Baldwin C, Puthucheary Z, Morris P, Mortimore J, Hart N, Denehy L, Granger CL (2017) Factors influencing physical activity and rehabilitation in survivors of critical illness: a systematic review of quantitative and qualitative studies. Intensive Care Med 43(4):531-542. https://doi.org/10. 1007/s00134-017-4685-4

11. Holdsworth C, Haines KJ, Francis JJ, Marshall A, O'Connor D, Skinner EH (2015) Mobilization of ventilated patients in the intensive care unit: an elicitation study using the theory of planned behavior. J Crit Care 30(6):1243-1250. https://doi.org/10.1016/j.jcrc. 2015.08.010

12. Hush JM, Cameron K, Mackey M (2011) Patient satisfaction with musculoskeletal physical therapy care: a systematic review. Phys Ther 91(1):25-36. https://doi.org/10.2522/ptj.20100061

13. Walker W, Wright J, Danjoux G, Howell SJ, Martin D, Bonner S (2014) Project Post Intensive Care eXercise (PIX): a qualitative exploration of intensive care unit survivors' perceptions of quality of life post-discharge and experience of exercise rehabilitation. J Intensive Care Soc 16(1):37-44. https://doi.org/10.1177/ 1751143714554896

14. Deacon KS (2012) Re-building life after ICU: a qualitative study of the patients' perspective. Intensive Crit Care Nurs 28(2):114-122. https://doi.org/10.1016/j.iccn.2011.11.008

15. Sottile PD, Nordon-Craft A, Malone D, Schenkman M, Moss M (2015) Patient and family perceptions of physical therapy in the medical intensive care unit. J Crit Care 30(5):891-895. https://doi. org/10.1016/j.jcrc.2015.04.119 
16. Burry L, Cook D, Herridge M, Devlin JW, Fergusson D, Meade M, Steinberg M, Skrobik Y, Olafson K, Burns K, Dodek P, Granton J, Ferguson N, Jacka M, Tanios M, Fowler R, Reynolds S, Keenan S, Mallick R, Mehta S, Investigators S, Canadian Critical Care Trials G (2015) Recall of ICU stay in patients managed with a sedation protocol or a sedation protocol with daily interruption. Crit Care Med 43(10):2180-2190. https://doi.org/10.1097/CCM. 0000000000001196

17. Schwarzkopf D, Behrend S, Skupin H, Westermann I, Riedemann NC, Pfeifer R, Günther A, Witte OW, Reinhart K, Hartog CS (2013) Family satisfaction in the intensive care unit: a quantitative and qualitative analysis. Intensive Care Med 39(6):1071-1079. https://doi.org/10.1007/s00134-013-2862-7

18. Jones C, Humphris G, Griffiths R (2000) Preliminary validation of the ICUM tool: a tool for assessing memory of the intensive care experience. Clin Intensive Care 11(5):251-255

19. U.S. National Library of Medicine (2017) How to write easy-toread health materials. https://medlineplus.gov/etr.html. Accessed 10 May 2018

20. Myhren H, Ekeberg O, Toien K, Karlsson S, Stokland O (2010) Posttraumatic stress, anxiety and depression symptoms in patients during the first year post intensive care unit discharge. Crit Care 14(1):R14. https://doi.org/10.1186/cc8870
21. Zetterlund P, Plos K, Bergbom I, Ringdal M (2012) Memories from intensive care unit persist for several years-a longitudinal prospective multi-centre study. Intensive Crit Care Nurs 28(3):159-167. https://doi.org/10.1016/j.iccn.2011.11.010

22. Legg AM, Andrews SE, Huynh H, Ghane A, Tabuenca A, Sweeny K (2015) Patients' anxiety and hope: predictors and adherence intentions in an acute care context. Health Expect 18(6):3034-3043. https://doi.org/10.1111/hex.12288

23. Jolley SE, Regan-Baggs J, Dickson RP, Hough CL (2014) Medical intensive care unit clinician attitudes and perceived barriers towards early mobilization of critically ill patients: a cross-sectional survey study. BMC Anesthesiol 14:84. https://doi.org/10.1186/1471-225314-84

24. Davidson JE, Harvey MA (2016) Patient and family post-intensive care syndrome. AACN Adv Crit Care 27(2):184-186. https://doi. org/10.4037/aacnacc2016132

25. Higgins T, Larson E, Schnall R (2017) Unraveling the meaning of patient engagement: a concept analysis. Patient Educ Couns 100(1): 30-36. https://doi.org/10.1016/j.pec.2016.09.002

Publisher's note Springer Nature remains neutral with regard to jurisdictional claims in published maps and institutional affiliations. 